\section{Clarifying nuclear structure}

\author{
Sorry, for copyright \\ reasons some images \\ on this page may not \\ be available online
}

The last chapter, occupying over half the book, is devoted to vibrational spectra. There is a full account of the quantal theory of harmonic vibrations, the normal modes of vibration and the sum rules for multipole oscillator strength. Sections follow on particlevibration coupling and anharmonicity in vibrational motion; there are also appendices on the liquid drop model of vibrations and rotations and on the five-dimensional quadrupole oscillator.

The level of presentation is such that it can be understood by a graduate student, and yet it is so comprehensive that most aspects of nuclear collective motion are covered. In a book of this kind there is a danger that the reader is overwhelmed by a mass of detail, and fails to see the essential theoretical structure underlying the specialised applications. Bohr and Mottelson

\& attempt to avoid this by dividing the

$\ddot{\%}$ book into three levels: text, illustrative

examples and appendices. The text contains the basic theory, presented in as straightforward and uncluttered a manner as possible, and its application to analyse the structure of particular nuclei is relegated to the second level. The appendices contain standard mathematical derivations and formulae. On the whole this division is successful in clarifying the presentation.

As would be expected, the treatment is thorough and detailed throughout, so that it will remain an indispensable work of reference for many years to come. $\quad$ P. E. Hodgson

quantisation certainly fills a gap in the earlier books. Scattering theory is better covered, and provides a logical introduction to the S-functional approach of Bogoliubov and to the perturbation expansion of Feynman and Dyson. This section ends with a brief account of renormalisation theory. There follows an account of the general results of the theory, usually in great detail but occasionally avoiding overlap with the other books by referring to them. Infinite-component fields and parastatistics are among new topics. The book ends with a very brief account of the $C^{*}$. algebra approach, and the progress of constructive field theory up to 1971 .

The bibliography is quite large and contains very useful references to Russian work on this subject. The book, being typed and photocopied, is rather bulky. It is well translated, authoritative and suitable as a companion and guide for postgraduate students in physics or mathematics studying relativistic quantum mechanics, and for other rescarch workers seeking a way in to this difficult subject. 